\title{
Comportamiento Morfoagronómico de Diez Familias Clónales de Papa (Solanum tuberosum L.) en las Condiciones Climáticas del Sur de Brasil ${ }^{1}$
}

\author{
Mario A Andreu ${ }^{2}$, Elianne Augustin ${ }^{3}, S$. \\ Arione da Pereira ${ }^{4}$
}

\begin{abstract}
Resumen
La introducción de nuevos cultivares de papa que presenten una buena adaptación y desempeño, es muy importante para las condiciones locales de la región sur del Brasil. Por lo tanto, en la EMBRAPA, de Clima Templado (CPACT), durante la primavera del 2000 y otoño del 2001, se condujeron una serie de experimentos que permitieron evaluar en condiciones de campo la respuesta de 10 familias clónales de papa. Un total de 800 clones fueron evaluados en primera generación clonal y 400 clones en segunda generación clonal. Este trabajo busca estimar la confiabilidad de la selección precoz de tubérculos con base en la apariencia general y evaluar la calidad y producción de las diferentes familias. Se observaron coeficientes de correlación significativos y de alta magnitud para textura, forma de los tubérculos y profundidad de las yemas entre periodos de cultivo y por lo tanto, es posible realizar una selección precoz más efectiva para estas características. Los resultados de este estudio sugieren que la selección para las características de calidad y cantidad de tubérculos es recomendada para los cultivos de primavera, donde las condiciones climáticas son más favorables y se consiguen maximizar estos parámetros. La apariencia general estuvo sujeta principalmente a las variaciones de las condiciones climáticas por lo que no se consideró como un parámetro efectivo para la selección precoz cuando los periodos de cultivos son contrastantes. De los cruzamientos evaluados sólo el cruzamiento C-1226-35-80 x ASTERIX demostró mayor flexibilidad fenotípica para peso por planta en los dos periodos de cultivo solo siendo superado por la cultivar ELVIRA. Las familias clónales sólo lograron un buen desempeño frente a los testigos en las

1 Trabajo perteneciente a Tesis de Graduación.

2 Licenciado en Genética. Facultad de Ciencias Exactas, Químicas y Naturales. Universidad Nacional de Misiones. Félix de Azara 1552. (3300) Posadas, Misiones, Argentina. Autor para correspondencia E-mail: marioandreu@hotmail.com.

3 Ph.D. Investigadora. EMBRAPA C.P.A.C.T. BR 392, Km 78, Caixa Postal 403, 96001-970 Pelotas, RS, Brasil. E-mail: augustin@cpact.embrapa.br.

4 Ph.D. Investigador. EMBRAPA C.P.A.C.T. BR 392, Km 78, Caixa Postal 403, 96001-970 Pelotas, RS, Brasil. E-mail: arione@cpact.embrapa.br
\end{abstract}


condiciones adversas de otoño, para número de tubérculos por planta, solo siendo superadas por el cultivar Macaca.

Palabras claves adicionales: Solanum tuberosum, selección precoz, fotoperíodo, temperatura, producción.

\section{Morphoagronomic Behaviour of Ten Clonal Potato (Solanum tuberosum) Families Under the Southern Brazil Climatic Conditions}

\section{Summary}

The introduction of new potato cultivars that have good adaptation and agronomic performance is very important for the local conditions of the southern region of Brazil. A series of experiments were carried out at EMBRAPA Clima Templado (CPACT) during the spring of 2000 and autumn of 2001, which allowed to evaluate the response of 10 hybrid populations to determine the confidence of early generations selection.

A total of 800 first generation clones and 400 clones of second clonal generation and four control cultivars were selected for tuber general appearance, quality and yield. High and significant correlation coefficients were observed for tuber texture, shape and eye depth ness between growing seasons. These results imply that early generation selection could be done for these traits during the spring, which present better environment conditions that maximize the parameters.

Tuber general appearance was affected by variations in environmental conditions and gave no effective response to early generations selection at contrasting growing conditions.

Cross C-1226-35-80 x Asterix presented higher phenotypic plasticity for tuber yield at both growing seasons and was out yielded only by cultivar Elvira. Clonal families had better performances in tuber yield than control cultivars only in the autumn season where environmental conditions are poorer and were out yielded only by cultivar Macaca.

Additional Index words:

Solanum tuberosum, early selection, Photoperiod, temperature, production.

\section{Introducción}

En Río Grande do Sul, la papa es cultivada en dos épocas del año 
climatológicamente contrastantes. Estas épocas corresponden a los meses de agosto - noviembre donde las condiciones son de fotoperíodo, temperatura y radiación crecientes, y los meses de febrero - junio donde se observa lo contrario, es decir, fotoperíodo, temperatura y radiación decrecientes (7).

El fotoperíodo y la temperatura son factores influyentes sobre el desempeño de los cultivares de papa, llegando a convertirse en una limitante para la producción de la planta en una determinada región. Días cortos y temperaturas medias o templadas estimulan la formación de los tubérculos, mientras que días largos y temperaturas altas restringen su formación (2).

Los cultivares de la región sur de Brasil se diferencian en producción de tubérculos en los diferentes periodos de cultivo, siendo los cultivares mejor adaptados más productivos en el periodo de primavera que en el de otoño (7). Por eso es importante la incorporación de cultivares que posean mayor flexibilidad fenotípica que permitan un mejor desempeño en ambos periodos.

Los programas de mejoramiento genético de la papa del EMBRAPA CPACT se inician con el cruzamiento de dos variedades (o clones). Esta población fue sometida a varios ciclos de selección en ambos periodos, procediendo posteriormente a la identificación de genotipos superiores.

El mejoramiento está casi siempre asociado a características heredadas cuantitativamente y, por lo tanto, bastante influenciados por el ambiente, dificultando la evaluación y discriminación de los mejores genotipos. A menudo se enfrenta el problema de no poderse seleccionar genotipos superiores debido a que las evaluaciones realizadas sobre los genotipos son de carácter subjetivo (1).

El desempeño de las plantas originadas por semillas verdaderas, no es el mismo que aquel obtenido de tubérculo semilla de tamaño comercial $>45$ $\mathrm{mm} \varnothing)$, lo que hace que la eficiencia de la selección en las 2 primeras generaciones clónales sea baja $(5 ; 9 ; 10 ; 11)$.

Este trabajo tuvo como objetivo determinar la confiabilidad de la selección temprana, en ambos periodos de cultivo con base en la estimación de la apariencia general y destacar los cruzamientos con mejor desempeño morfoagronómico en ambas generaciones clónales.

\section{Materiales y Métodos}

Los trabajos fueron iniciados con un grupo de 10 familias clonales de papa pertenecientes al Programa de Mejoramiento de Papa de la EMBRAPA en el ambiente de clima templado de Pelotas, RS, Brasil, que fueron evaluados en primera y segunda generación clonal. Los experimentos fueron conducidos en Pelotas $\left(32^{\circ} 41^{\prime} \mathrm{S}\right)$, Brasil. El suelo utilizado fue clasificado como Podzólico Rojo - Amarillo Eutrófico y abonado con $2000 \mathrm{~kg} / \mathrm{ha}^{-1}$ de NPK (5- 
20-10) en primera generación clonal y (10-20-10) en segunda generación clonal. La apertura de los surcos, la siembra y cosecha fueron manuales, y las labores culturales y fitosanitarias se realizaron según las instrucciones técnicas de la EMBRAPA en un ambiente de clima templado. El distanciamiento fue de $80 \mathrm{~cm}$ entre surcos y $30 \mathrm{~cm}$ entre plantas.

Clones Primera Generación. 800 clones de papa fueron evaluados. Los tubérculos provenían de semilla verdadera de papa llevada de invernadero a campo para ser multiplicadas. Se usaron las combinaciones de progenitores: ATLANTIC x MACACA, CRISTAL x ATLANTIC, C-1485-6-87 x ATLANTIC, C-1226-35-80 x ASTERIX, DAYSI x MACACA, BARONESA x MONALISA, MONTE BONITO $\times$ ASTERIX, LADY ROSETA $x$ MACACA, CRISTAL $x$ MONALISA, AGRIA x BARONESA y cuatro controles ATLANTIC, MACACA, BARONESA y ELVIRA. El diseño experimental consistió en bloques completamente al azar de 11 parcelas, con 20 plantas por parcela y 4 repeticiones.

Clones Segunda Generación. Se evaluaron 400 clones seleccionados de primera generación clonal y cuatro controles (ATLANTIC, BARONESA, MACACA, ELVIRA) distribuidos en 104 parcelas con 4 repeticiones, con un delineamento de bloques al azar con parcelas de 5 plantas.

Los datos de los clones tomados en los dos experimentos fueron: número y peso promedio de tubérculos por planta, índice de forma de los tubérculos derivado de la relación ancho / largo x 100: redonda (1) - oval (2) - oval alargada (3) - alargada (4); Textura: Lisa (1) - Áspera (2) - Reticulada (3); Profundidad de las Yemas: Sobresalientes (1) - Superficiales (2) - Semiprofundas (3) - Profundas (4); Apariencia General del tubérculo: (1 = Mala -4 = Muy Buena). Fueron estimados los análisis de variancia, medias, coeficientes de variación y coeficientes de correlación entre las diferentes épocas de cultivo.

\section{Resultados y Discusión}

Las medias de las características analizadas presentaron diferencias entre las épocas de cultivo, principalmente para el número y peso medio de tubérculos por planta, textura y apariencia general, a excepción de la forma de los tubérculos y profundidad de las yemas (Tabla 1).

Las condiciones climáticas subtropicales favorecen estas diferencias debido a que, estos periodos contrastantes de cultivo presentan diferentes niveles de temperatura, fotoperíodo y radiación (6). Las condiciones de cultivo de primavera, generalmente inducen a la formación de tubérculos mayores acompañados de un aumento en número de los mismos (3). El cultivo de otoño se destacó por la presencia de plantas con un menor número de tubérculos por planta acompañados de un menor peso promedio debido a un fotoperíodo más corto acompañado de una reducción del área foliar, con

una consiguiente tasa productiva más baja que en primavera (8). Durante el 
cultivo de primavera, de todas las familias clónales analizadas, el único cruzamiento que consiguió sobresalir fue C-1226-35-80 x ASTERIX para la característica peso medio de tubérculos por planta, mas no consiguió superar al cultivar testigo ELVIRA. Para el número medio de tubérculos por planta ningún cruzamiento consiguió superar a las cultivares ELVIRA y BARONESA, aunque el cruzamiento LADY ROSETA x MACACA superó a las demás familias y a los otros testigos. Esto no se repite durante el año siguiente donde las medias de las familias y de los testigos exhiben una reducción en sus valores y no poseen un buen desempeño productivo. Las familias clónales en general, consiguen igualar la media para tubérculos por planta del cultivar más productivo que fue MACACA. Sin embargo, el cultivar ELVIRA continuó con el mayor peso por planta seguida por el cruzamiento C-1226-35-80 (Figuras 1 y 2). La textura presentó una media de tubérculos de piel áspera durante la primavera, principalmente debido a la gran cantidad de genotipos de diferentes estados en campo con mayor o menor susceptibilidad a los cambios ambientales y del suelo que afectan a esta característica. Una vez que los clones indeseados en cuanto a la piel áspera son eliminados, se consigue con relativa eficiencia en la generación siguiente una media general más cercana a la piel de aspecto liso. La forma y profundidad de las yemas presentaron medias semejantes en las condiciones de cultivo de primavera y otoño, correspondiendo a tubérculos con una forma predominantemente oval-alargada y yemas con profundidad semi-superficial.

Tabla 1. Coeficientes de correlación de los cultivos de primavera del 2000 y otoño de 2001 para las características morfoagronómicas de los clones de papa estudiados. EMBRAPA - CPACT.

\begin{tabular}{|l|c|c|c|c|c|c|c|}
\hline \multicolumn{1}{|c|}{ Característica } & $\mathbf{2 0 0 0}$ & $\mathbf{2 0 0 1}$ & $\mathbf{2 0 0 0}$ & $\mathbf{2 0 0 1}$ & $\begin{array}{c}\mathbf{2 0 0 0} \\
\mathbf{( \% )}\end{array}$ & $\begin{array}{c}\mathbf{2 0 0 1} \\
\mathbf{( \% )}\end{array}$ & $\begin{array}{c}\text { Coef. De } \\
\text { corre- } \\
\text { lación }\end{array}$ \\
\hline $\begin{array}{l}\text { No. de tubérculos } \\
\text { por planta }\end{array}$ & 7.10 & 6.38 & $4.44-14$ & $2-15$ & 28.07 & 22.65 & $0.140^{\star *}$ \\
\hline $\begin{array}{l}\text { Peso de tubérculos } \\
\text { por planta }\end{array}$ & 442.3 & 271.07 & $227-958$ & $53-644$ & 32.21 & 31.62 & $0.376^{\star \star}$ \\
\hline Textura & 2 & 1 & $1-3$ & $1-3$ & 19.40 & 25.37 & $0.628^{\star \star}$ \\
\hline Forma & 3 & 3 & $1-4$ & $1-4$ & 28.67 & 16.08 & $0.748^{\star \star}$ \\
\hline $\begin{array}{l}\text { Profundidad de } \\
\text { yemas }\end{array}$ & 3 & 3 & $1-4$ & $1-4$ & $\begin{array}{c}12.93 \\
14.44\end{array}$ & $0.735^{\star *}$ \\
\hline Apariencia general & 3 & 2 & $2-4$ & $1-4$ & $\begin{array}{c}12.91 \\
\%\end{array}$ & $\begin{array}{c}32.78 \\
\%\end{array}$ & -0.072 \\
\hline
\end{tabular}

**Significativo a $p<0,01$ por la prueba t. 


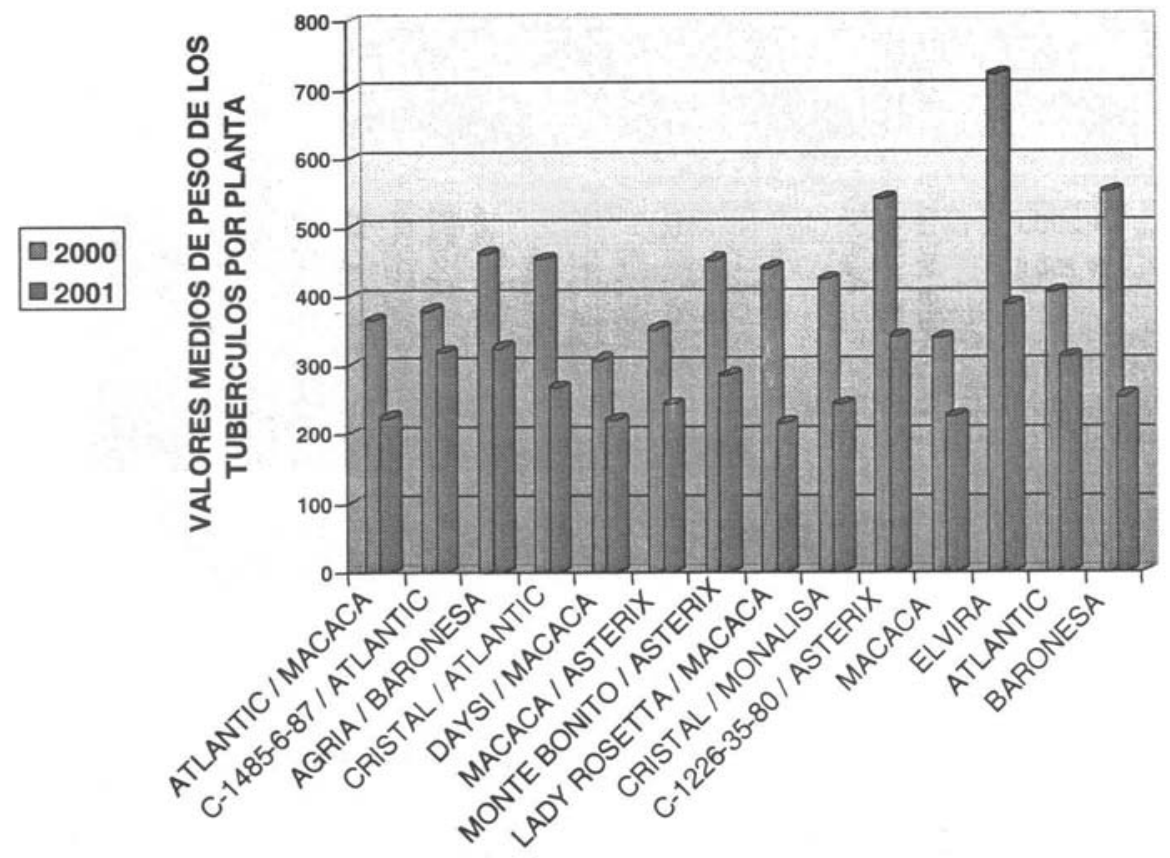

FAMILIAS Y TESTIGOS

Figura 1. Comparación de los valores medios para peso de los tubérculos por planta entre las familias analizadas y los testigos en la primavera del año 2000 y otoño del 2001. EMBRAPA - CPACT.

Los coeficientes de variación, son indicativos de la variación fenotípica y de la amplia variabilidad existente para todas las características, siendo mayores para número medio de tubérculos por planta y forma de los tubérculos en el año 2000 y para peso medio por planta y apariencia general en el año 2001. Los menores coeficientes de variación fueron para peso medio de tubérculos por planta y apariencia general en el año 2000 y profundidad de las yemas en el 2000 y 2001 respectivamente. Esto sugiere que puede aplicarse una selección más efectiva en los periodos de primavera para número medio de tubérculos por planta y forma de los tubérculos (7). 


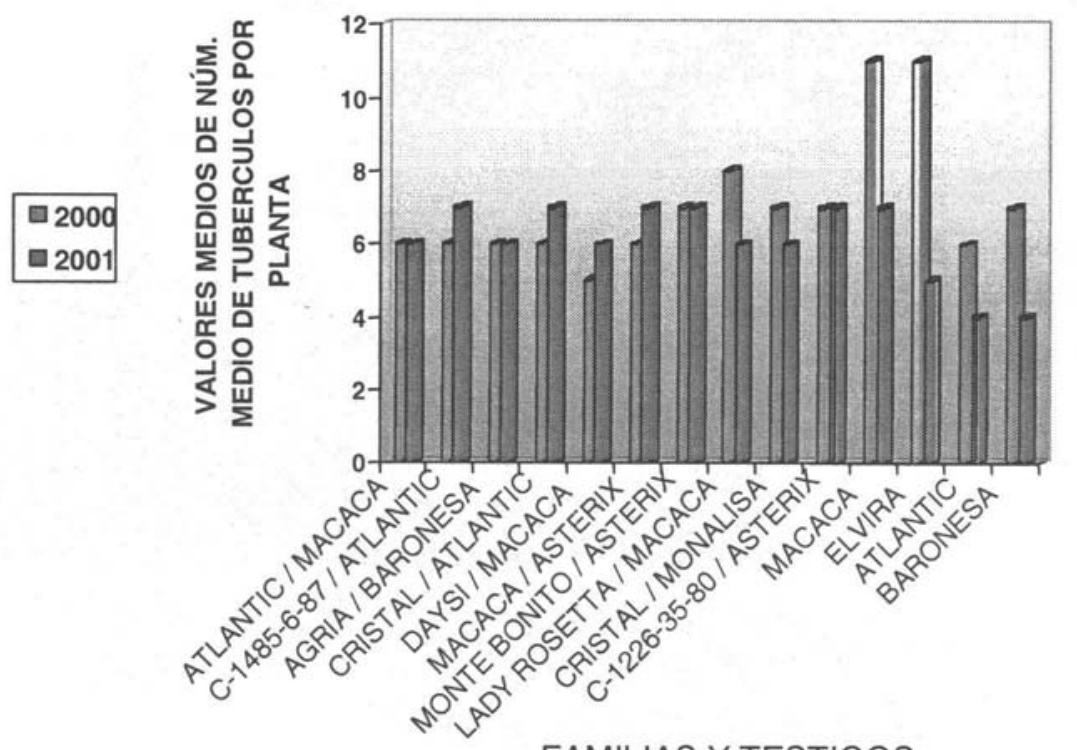

\section{FAMILIAS Y TESTIGOS}

Figura 2. Comparación de los valores medios para número de tubérculos por planta entre las familias analizadas y los testigos en el cultivo de primavera del año 2000 y otoño del 2001. EMBRAPA - CPACT.

Los coeficientes de correlación entre los periodos de cultivo fueron significativos excepto para la apariencia general, y de baja magnitud para número de tubérculos por planta, peso medio de los tubérculos y la apariencia general, a diferencia de los altos coeficientes de correlación observados para textura, forma y profundidad de las yemas, indicando que a pesar de las diferencias ambientales y la diversidad genética evaluadas, estos caracteres pueden sufrir intensidades de selección mayores en las generaciones tempranas de un programa de mejoramiento (4). Estas diferencias en los coeficientes de correlación observadas son debidas tanto a factores ambientales como genéticos de los materiales utilizados.

Las magnitudes de las correlaciones indican que independientemente de las condiciones de cultivo, el número y peso de tubérculos, junto con la apariencia general, son parámetros que exigen una intensidad de selección baja para no perjudicar las poblaciones posteriores formadas a partir de ellas. 


\section{Conclusiones}

- De todos los cruzamientos evaluados solamente C-1226-35-80 x ASTERIX demostró mayor flexibilidad fenotípica para peso por planta en los dos periodos de cultivo solo siendo superado por el cultivar ELVIRA.

- A pesar de las condiciones adversas de otoño, las familias clónales en general consiguieron igualar el desempeño de los cultivares testigos para número de tubérculos por planta, siendo sólo superadas por el cultivar MACACA.

- Las características de textura, forma de los tubérculos y profundidad de las yemas están altamente correlacionas entre los periodos de cultivo, por lo tanto, es posible realizar una selección precoz más efectiva para estas características.

- La apariencia general estuvo sujeta principalmente a las variaciones de las condiciones climáticas que predominaron en las épocas de cultivo, presentando una media inferior para las características cualitativas y cuantitativas de los tubérculos en el periodo de otoño. Por lo tanto, no se considera como un parámetro efectivo para la selección precoz cuando los periodos de cultivos son contrastantes.

- La selección para calidad y cantidad de tubérculos es recomendada para los cultivo de primavera, donde las condiciones climáticas son más favorables y se consiguen maximizar estos parámetros.

\section{Referencias Bibliográficas}

1. Barbosa, M.H.P., C.A.B.P. Pinto. 1988. Eficiencia de índices de seleção na identificação de clones superiores de batata. Pesquisa Agropecuaria Brasilera, 33 (2):149 -156.

2. Beukema, H.P., D.E. van der Zaag. 1990. Introduction to potato production. Wageningen: Centre for Agricultura Publishing Documentation (PUDOC), $208 \mathrm{p}$.

3. Costa, D.M. da, N.F. Lopes. 1981. Período e Velocidade de Tuberização em Cinco Cultivares de Batata (Solanum tuberosum L.). Revista Ceres, 28 : $530-545$.

4. Gopal, J., P.C. Gaur, M.S. Rana. 1992. Early generation selection for agronomic characters in a potato breeding programme. Theoretical and Applied Genetics, 84:709 - 713. 
5. Gopal, J. 1997. Progeny Selection for Agronomic Characters in Early Generation of a Potato Breeding Program. Theoretical and Applied Genetics, New York, 95: 307 -311.

6. Lemaga, B., K. Caesar. 1990. Relationships between numbers of main stems and yield components of potato (Solanum tuberosum L. CV. Erntestolz) as influenced by different day lengths. Potato Research, Wageningen, 33: 257-267.

7. Pereira, A da S. 1999. Correlações entre cultivo de primavera e de outono para algumas características agronómicas em batata. Agropecuaria Clima Temperado, Pelotas, 2 (2): 207-212.

8. Pereira, A da S., A. Campos. 1999. Teor de Açúcar em Genotipos de Batata (Solanum tuberosum L). Ciencia Rural, Santa Maria, 29 (1):13-16.

9. Pinto, C.A.B.P., V.I.R. Valverde, M.S. Rossi. 1994. Eficiencia de Seleção nas Primeiras Gerações Clonais da Batata (Solanum tuberosum L). Pesquisa Agropecuaria Brasileira, 29 (5):771-778.

10. Pinto, C.A.B.P. 1999. Melhoramento Genético da Batata. Informe Agropecuario, Belo Horizonte, 20 (147):120 -128.

11. Tai, G.C.C., D.A. Young. 1984. Early Generation Selection for Important Agronomic Characteristics in Potato Breeding Population. American Potato Journal, 61: 419 - 439. 